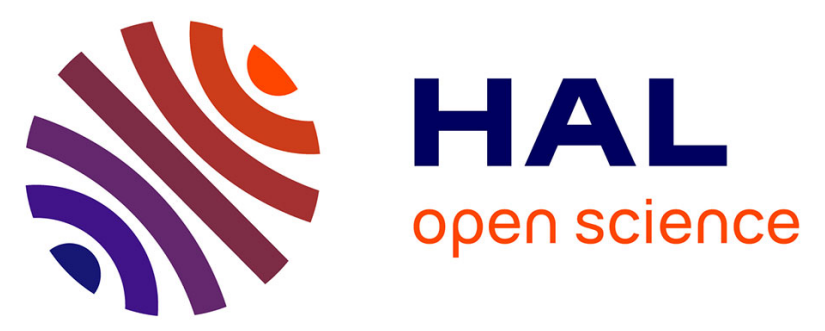

\title{
A Lack of Clinical Effect of High-frequency rTMS to Dorsolateral Prefrontal Cortex on Bulimic Symptoms: A Randomised, Double-blind Trial
}

Aurélia Gay, Isabelle Jaussent, Torrance Sigaud, Stéphane Billard, Jérôme Attal, Maude Sénèque, Bogdan Galusca, Frederique van den Eynde, Massoubre Catherine, Philippe Courtet, et al.

\section{To cite this version:}

Aurélia Gay, Isabelle Jaussent, Torrance Sigaud, Stéphane Billard, Jérôme Attal, et al.. A Lack of Clinical Effect of High-frequency rTMS to Dorsolateral Prefrontal Cortex on Bulimic Symptoms: A Randomised, Double-blind Trial. European Eating Disorders Review, 2016, 24 (6), pp.474-481. 10.1002/erv.2475 . inserm-01382734

\section{HAL Id: inserm-01382734 https://www.hal.inserm.fr/inserm-01382734}

Submitted on 17 Oct 2016

HAL is a multi-disciplinary open access archive for the deposit and dissemination of scientific research documents, whether they are published or not. The documents may come from teaching and research institutions in France or abroad, or from public or private research centers.
L'archive ouverte pluridisciplinaire HAL, est destinée au dépôt et à la diffusion de documents scientifiques de niveau recherche, publiés ou non, émanant des établissements d'enseignement et de recherche français ou étrangers, des laboratoires publics ou privés. 


\title{
A lack of clinical effect of high-frequency rTMS to dorsolateral prefrontal cortex on bulimic symptoms: a randomised, double-blind trial
}

\author{
A GAY ${ }^{\mathrm{a}, \mathrm{b}}$, I JAUSSENT, T SIGAUD $^{\mathrm{a}, \mathrm{b}}, \mathrm{S} \mathrm{BILLARD}^{\mathrm{a}}, \mathrm{J}^{\mathrm{ATTAL}}{ }^{\mathrm{c}, \mathrm{d}}, \mathrm{M} \mathrm{SENEQUE}^{\mathrm{c}, \mathrm{e}}, \mathrm{B}$ \\ GALUSCA $^{\mathrm{b}}, \mathrm{F}_{\text {VAN DEN EYNDE }}^{\mathrm{f}}, \mathrm{C}^{\mathrm{C}}$ MASSOUBRE $^{\mathrm{a}, \mathrm{b}}, \mathrm{Ph} \mathrm{COURTET}^{\mathrm{c}, \mathrm{e}} \& \mathrm{~S}$ \\ GUILLAUME ${ }^{*, e}$
}

a University Hospital of St-Etienne, Department of Psychiatry and Addictology, North Hospital, St Etienne, France

b TAPE Research Team, Jean Monnet University, Saint Etienne, France

cInserm U1061, University of Montpellier, Montpellier, France

d University Department of Adult Psychiatry, CHU Montpellier, Montpellier, France

e Department of Psychiatric Emergency \& Acute Care, Lapeyronie Hospital, CHRU Montpellier, Montpellier, France

f McGill University, Department of Psychiatry, Douglas Hospital, Eating Disorders Program, 6875 Blvd LaSalle, Montreal, Canada

${ }^{*}$ Correspondence to:Sébastien Guillaume, Department of Psychiatric Emergency \& Acute Care, Hospital Lapeyronie, Avenue Gaston Giraud, 34295 Montpellier cedex 5, France

Phone: +33 467338 581; Fax: +33467338988

E-mail: s-guillaume@chu-montpellier.fr

FUNDING SOURCE AND FINANCIAL DISCLOSURES

This study received financial support from CHRU Montpellier (PHRC-IR UF 8709). The CHRU Montpellier had no role in the design, analysis, interpretation, or publication of this study. All authors declare they have no conflict of interest related to this study.

\section{Word counts:}

Abstract: 149

Text: 3602 


\begin{abstract}
Studies suggests that stimulation of the left dorsolateral prefrontal cortex (DLPFC) reduces food craving in bulimic patients, but evidence supporting rTMS as a therapeutic tool is lacking.We investigated the safety and therapeutic efficacy of an adjunct high-frequency rTMS program targeting the left DLPFC. Forty-seven females with bulimia nervosa were randomised to a real or sham stimulation group. The real group underwent ten rTMS sessions, each consisting of 20 trains of 5 seconds with 55 -second intervals between trains, at a frequency of $10 \mathrm{~Hz}$. The main outcome was the number of binge episodes in the 15 days following the end of stimulation. Overall, no significant improvement in binging and purging symptoms was noted after the program. rTMS was well tolerated. This suggests that ten sessions of high-frequency rTMS to the left DLPFC provide no greater benefit than placebo. Future studies should consider methodological issues as well as alternative targets.
\end{abstract}

Key words: eating disorders, bulimia nervosa, neuromodulation, craving 


\section{Introduction}

Eating disorders (EDs) are serious mental illnesses (Klump, Bulik, Kaye, Treasure, \& Tyson, 2009). The data indicate that the overall prognosis for individuals with bulimia nervosa $(\mathrm{BN})$ remains unsatisfactory, with less than half the patients attaining full remission and about a quarter experiencing a chronic course (Steinhausen \& Weber, 2009). Mortality is increased in BN, especially by suicide (Standardized Mortality Ratio: 6.9) (Crow et al., 2009). Moreover, EDs affect the quality of life of both the patients and their families (Zabala, Macdonald, \& Treasure, 2009), and individuals with these disorders have particularly high rates of health service use (Mitchell et al., 2009; Striegel-Moore et al., 2008).

The most frequent treatment for $\mathrm{BN}$ is the cognitive behavioural therapy program especially developed for EDs (Fairburn, Cooper, \& Shafran, 2003). Nevertheless, the posttreatment results remain unsatisfactory in terms of remission, with an end to binge-eating episodes achieved in only 37\% of BN patients (Hay, Bacaltchuk, Stefano, \& Kashyap, 2009). Other psychotherapies have been proposed and the most frequently proposed pharmacological alternative is serotonergic antidepressants, which at least improve the medium-term symptomatology, but again not enough (Treasure, Claudino, \& Zucker, 2010). Thus, there is an indisputable need for novel, brain-directed adjuncts to treat $\mathrm{BN}$.

Repetitive transcranial magnetic stimulation (rTMS) is now widely used in a variety of neurological and psychiatric disorders. A growing body of data suggests that high-frequency stimulation of the left dorsolateral prefrontal cortex (DLPFC) decreases craving in smoking, alcoholism and cocaine addiction (for review (Grall-Bronnec et al., 2015; Jansen et al., 2013)). BN-individuals display aberrant patterns of prefrontal cortex activity during wide range of experimental task and $\mathrm{BN}$ are assumed to have an addictive component (for rewiew see Hadad et al 2014(Hadad \& Knackstedt, 2014)). There are also evidences that food craving is associated with DLPFC activity (Kekic et al., 2014) and previous study found that the 
capacity for self-control depends on DLPFC activity levels(Hare, Camerer, \& Rangel, 2009)Thus, it is possible that rTMSof the DLPFC could reduce overeating behaviours by reducing food cravings and by improving cognitive control. The impact of one session of rTMS on food craving was assessed in healthy subjects with high levels of craving (Uher et al., 2005) and in subjects with BN or other binge-eating disorders (Van den Eynde et al., 2010). These studies showed that high-frequency stimulation of the left DLPFC reduced food craving. In addition, the anxiety induced by viewing food stimuli (measured by salivary cortisol) was significantly decreased after rTMS in young women with BN (Van den Eynde et al., 2010). Furthermore, in a recent study using near-infrared spectroscopy in eight patients with $\mathrm{BN}$, a significant decrease in cerebral oxygenation of the left DLPFC was also observed after a single session of rTMS during cognitive tasks measuring self-regulatory control in response to food and photo stimuli (Sutoh et al., 2016). Yet solid evidence of rTMS as a therapeutic tool in bulimia is lacking. Only one randomised controlled trial with repeated sessions has been published thus far. This study assessed the effects of 15 sessions of rTMS over three weeks in BN patients (Walpoth et al., 2008). The authors found no significant difference between the two groups. However, this negative result might be related to the lack of power due to the small sample size ( $n=14,7$ per group).

We performed a randomised controlled trial in a large sample of SSRI-resistant BN patients to assess the effect of a two-week program of high-frequency rTMS on the left DLPFC and the bulimic symptoms in the following weeks. We hypothesized that an rTMS program would significantly decrease the number of binge episodes compared with a sham group. 


\section{Material and Methods}

\section{$\underline{\text { Study design and participants }}$}

Over a two-year period, we screened female patients with a current diagnosis of bulimia nervosa followed in the outpatient departments in two academic centres in France (Montpellier and Saint Etienne). Inclusion criteria were BN for at least six months according to DMS-IV criteria, right-handed for safety reasons (Van den Eynde et al., 2010), no improvement under SSRI treatment at adequate and stable dose (i.e. 40 to $60 \mathrm{mg}$ of Fluoxetine) for at least five weeks, and age between 18 and 40 years.

Exclusion criteria were contraindication to rTMS determined by the Adult Safety Screen Questionnaire(Rossi, Hallett, Rossini, \& Pascual-Leone, 2009), previous exposure to rTMS, ongoing psychotherapy for BN (e.g. current CBT), pregnancy as ruled out by a pregnancy test, and refusal to use effective contraception for the entire rTMS program. Diagnosis was made with the eating disorders module of the Structured Clinical Interview for DSM-IV, Axis I Disorders/Patient Version. The study was approved by the local ethics committee (CPP SudMéditerranée IV) and written informed consent was obtained from all participants. The study has been registered in a clinical trial database (ClinicalTrials.gov Identifier: NCT01530906).

The participants were evaluated at baseline using open questions to assess the eating disorder history and features (number of binges, number of vomiting episodes, etc.) over the previous 15 days. In addition, EDs were assessed with the Eating Disorder Inventory 2 (EDI2) (Gardner, 1991). This six-point self-report questionnaire assesses specific attitudes or behaviours concerning eating, weight and body shape, bulimia, and personality traits like perfectionism and body dissatisfaction. Lifetime Axis I psychiatric diagnoses were made according to the DSMI-IV, using the French version of the lifetime Mini International Neuropsychiatric Interview (Sheehan et al., 1998), and current mood state was assessed using 
the Montgomery-Asberg Depression Rating Scale (MADRS).

After the initial visit, participants were randomly assigned to either the real rTMS or the sham rTMS group. The randomisation was stratified by centre and was prepared with random blocks of four participants. In each centre, two researchers were in charge of the protocol, with one conducting the rTMS and the other performing the clinical assessment. The latter researcher and the participants were blind to the rTMS (real or sham) allocation. In the two consecutive week following baseline assessment, the participants received ten sessions of real or sham rTMS (from Monday to Friday). The number of sessions was based on a study focussing in cigarette consumption with similar design(Amiaz, Levy, Vainiger, Grunhaus, \& Zangen, 2009). After each session, they were screened for safety issues and at sessions 1 and 10 they were asked to guess whether they had received real or sham rTMS. Between 15 and 21 days after the end of the rTMS sessions, they were reassessed for behaviour over the previous 15 days. The final assessment included the number of binges and other purging behaviours in the last 15 days, the maximum intensity of craving in the episode judged by the patient as the most important, and mood (by MADRS).

\section{$\underline{\text { rTMS procedure }}$}

Trained psychiatrists performed the stimulation with a figure-of-eight air-cooled coil (focal coil) attached to a rapid Magstim Stimulator (Magstim Company Ltd., Whitland, UK) placed over the left dorsolateral prefrontal cortex. Theresting motor threshold (RMT) was identified as the minimum magnetic field strength required to produce left thenar muscle visual activation by single TMS pulses delivered to the contralateral motor cortex for at least five of the ten trials. The coil placement for the defined DLPFC stimulation was $6 \mathrm{~cm}$ anterior to the motor cortex hotspot (Ahdab, Ayache, Brugieres, Goujon, \& Lefaucheur, 2010). The stimulation site was outlined on a cap, which was repositioned at each rTMS session. Based on previous research (McClelland, Bozhilova, Nestler, et al., 2013; Uher et al., 2005; Van den 
Eynde et al., 2010), each rTMS session consisted of 20 trains of 5 seconds with 55-second intervals between trains, at a frequency of $10 \mathrm{~Hz}$ and a motor threshold intensity of $110 \%$, for a total of 1000 pulses per session in 20 minutes. Sham treatment was delivered just like active treatment, differing only by the use of a specially designed sham.

\section{$\underline{\text { Outcome measures }}$}

The main outcome was the number of binge episodes in the 15 days before the final visit. The secondary outcomes were related to the characteristics of the binge episodes and the number of vomiting episodes before the final visit, the mood at the final visit, and the modification in the outcomes before and after the baseline and final assessments.

\section{$\underline{\text { Statistical analysis }}$}

The sample is described using percentages for categorical variables and medians and range for quantitative variables; the latter were mostly skewed according to the Shapiro-Wilk test. Demographic and clinical characteristics were compared between the sham and real rTMS groups using the Chi-square test or Fisher's exact test for categorical variables and the Mann-Whitney test for continuous variables (Tables 1 \& 2). Wilcoxon's signed-rank test compared repeated measurements (measurements prior to rTMS and 15 days after rTMS in each group). Both intention-to-treat and per-protocol analyses for the primary outcome (binge episodes) were conducted. The analysis in intention to treat used last observation carried forward and the analysis in per protocol took account only patients who complete the entire clinical trial. Significance level was set at $\mathrm{p}<0.05$. Analyses were performed with SAS statistical software (version 9.4; SAS Inc., Cary, NC, USA). 


\section{Results}

Participant screening and selection (Figure 1)

One hundred and six patients were approached after screening in our active file or responded to our advertisement. Among them, 51 were included (24 in Montpellier and 27 in Saint Etienne), 21 did not meet the inclusion criteria, 18 met the non-inclusion criteria, and 16 refused to participate. Five participants had BMI below $17.5 \mathrm{~kg} / \mathrm{m}^{2}$ but met all the BN criteria and were thus recruited for the study. Patients were randomly allocated to either the real rTMS ( $n=26)$ or sham rTMS ( $n=25)$ group. Four participants did not begin the study: two withdrew their consent and two were excluded because technical problems with the rTMS machine made it impossible to carry out the protocol.

Thus, 47 participants were included in the analysis. Nevertheless, five participants were lost to follow-up ( 2 in the sham and 3 in the rTMS group): one after the inclusion visit, one after the first rTMS session, one after the ninth session, and two after the tenth session.

\section{Baseline characteristics (Table 1)}

At baseline, the two groups were not statistically different for any of the demographic or ED characteristics, with the exception of a borderline difference for alcohol/substance abuse and perfectionism ( $\mathrm{p}=0.05$ for these two comparisons). The participants were also not significantly different regarding other lifetime history of comorbid psychiatric disorders and current mood state. The mean duration of the disease in the entire population was six years (0$25)$ and more than half of the participants (55\%) had previously been diagnosed with anorexia. $\underline{\text { Between group comparison at final visit }}$ (Table 2)

The two groups showed no significant differences for either the primary outcome (number of binges in the 15 days before the final visit) or the secondary outcomes (features of binge episodes, number of days without binging, maximal craving before a binge, number of vomiting episodes, mood). The results remained unchanged in per-protocol or intention-to- 
treat analyses and also remained similar after participants with BMI below 17.5 were excluded from analysis.

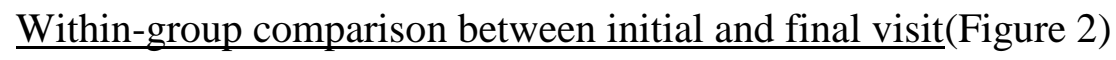

No significant improvement between the initial and final visit was observed in either group for the number of binge episodes $(\mathrm{p}=0.49$ for sham group and $\mathrm{p}=0.57$ for real $\mathrm{rTMS}$ group) or for the number of vomiting episodes $(\mathrm{p}=0.21$ for sham group and $\mathrm{p}=0.07$ for real rTMS group). MADRS showed a borderline significant decrease, indicating an improvement in mood, in the real rTMS group only $(\mathrm{p}=0.05)$. The results remained unchanged in perprotocol and intention-to-treat analyses and also remained unchanged after participants with BMI below 17.5 were excluded from analysis.

\section{$\underline{\text { Safety }}$}

Overall, the tolerance was good. Three participants experienced safety issues during the trial. One participant in the sham group made a suicide attempt between the end of rTMS and the final visit (related to an acute adverse life event). Another participant in the sham group and one in the real group experienced headaches after respectively session 3 and session 8 . The headaches responded to painkillers and these two participants completed the entire protocol.

\section{$\underline{\text { Blinding efficacy }}$}

Questions to determine the blinding efficacy revealed that only three of the 24 patients in the sham group and five of the 23 in the real rTMS group guessed their allocation correctly with a level of certitude above $50 \%$, either in session one or in session ten suggesting the good efficacy of blinding. 


\section{Discussion}

Our results suggest that the addition of ten sessions of high-frequency rTMS to the left DLPFC in patients with bulimic symptoms treated by adequate doses of SSRI does not yield greater benefits than placebo. Our results also suggest that rTMS is safe and well-tolerated in these patients. This agrees with the findings of the only small, randomised controlled trial focused on clinical outcomes (Walpoth et al., 2008).

Most studies in the addiction field that have investigated rTMS have evaluated its impact on craving and not on substance use or eating behaviour. Noninvasiveneuromodulation using rTMS has been found to be effective in reducing craving in several types of substance dependence (nicotine, alcohol, cocaine and methamphetamine) and eating disorders (food craving, Anorexia, BN and EDs not otherwise specified) with a medium effect size, particularly in bulimic disorders (Grall-Bronnec \& Sauvaget, 2014; Jansen et al., 2013; Sauvaget et al., 2015). Nevertheless, it can be hypothesized that the changes in craving induced by neuromodulation of the left DLPFC are not sufficient to have a clinical impact. Indeed, the data in the addiction literature are heterogeneous about the extent to which craving is associated with measures of drug-use behaviour or predictive of relapse. Also, studies are still needed to identify the conditions under which craving is more strongly associated with drug use (Tiffany \& Wray, 2012).

Another factor that might need to be considered is the variability in brain activity related to metabolic state, which would affect the resting brain state and the neuromodulatory response. Patients with $\mathrm{BN}$ usually follow restrictive diets that could have substantial effects on brain excitability and the sensitivity/response to neuromodulation(Val-Laillet et al., 2015). Furthermore, bias in sham stimulation has been suggested as being sometimes perceptible in rTMS(Broadbent et al., 2011). For example, (Barth et al., 2011) found that with an improved sham control condition, prefrontal rTMS inhibited food cravings no better than sham rTMS. 
The good blinding efficacy might explain the lack of difference between our two groups.

An alternative hypothesis is that methodological problems affect the results. For example, we cannot rule out that the absence of neuronavigation had an impact on our results, even though only one study in the addiction field (Herremans \& Baeken, 2012) has used this targeting method. MRI-based neuronavigation is much more precise and has been shown to improve the therapeutic effects on diseases like depression (Fitzgerald, Hoy, Daskalakis, \& Kulkarni, 2009; Nauczyciel et al., 2011; Schonfeldt-Lecuona, Cardenas-Morales, Freudenmann, Kammer, \& Herwig, 2010), auditory hallucinations (Klirova et al., 2013) and pain (Ahdab et al., 2010). Also, the participants in our protocol had ten rTMS sessions. The therapeutic benefit in depression was found to be higher for a higher number of sessions (Lam, Chan, Wilkins-Ho, \& Yatham, 2008) or for more than ten sessions (Gershon, Dannon, \& Grunhaus, 2003). Multiple rTMS sessions per day may also be a promising therapeutic development, as recently shown in depression (Baeken et al., 2013). Also we have only used behavioral measures that may be not sensitive enough. It would have been interesting to add some neuroimaging measure more sensitive to change. Regarding the stimulation parameters, we used high-frequency stimulation $(10 \mathrm{~Hz})$ and a suprathreshold intensity (110\% of RMT), with a total of 1000 pulses per session, in line with most studies in the field of addictive and eating disorders (Grall-Bronnec \& Sauvaget, 2014; Hone-Blanchet, Ciraulo, Pascual-Leone, $\&$ Fecteau, 2015); these last two parameters are thought to be predictive rTMS response factors in depression (Gershon et al., 2003; Lee, Blumberger, Fitzgerald, Daskalakis, \& Levinson, 2012; Mantovani et al., 2012; McDonald et al., 2011). It should also be noted that our sample was made up of patients with severe disorders resistant to a validated treatment for BN (i.e. fluoxetine) and/or with long-lasting disorders, and more than half of them had previous diagnoses of anorexia nervosa. Given that these last two characteristics are markers of poor therapeutic response (Eddy et al., 2007), it would be interesting to assess a less severe 
population in future studies, as has usually been done in studies assessing the efficiency of SSRIs to treat bulimia (Romano, Halmi, Sarkar, Koke, \& Lee, 2002). Finally, the absence of associations may be related to the effect size and we cannot exclude a lack a statistical power. Nevertheless, based on the present results, with a mean binge episodes difference between the two groups equal to 5.33 , a common SD equal to 14.31 , a power of $80 \%$, a type I error of 0.05 , 115 patients per group would needed to expect a significant difference between groups.

We targeted the left DLPFC in this study, but alternative targets should be considered for bulimic disorders. The right DLPFC has rarely been chosen in the addiction field; however, a recent meta-analysis of eight studies focused only on substance addiction found that active rTMS of the right DLPFC showed better results than sham (Enokibara, Trevizol, Shiozawa, \& Cordeiro, 2016). Another target of particular interest that is accessible to rTMS is the dorsomedial prefrontal cortex (DMPFC). The DMPFC plays an important role in selfregulation, including self-suppression of emotional response and impulse control. Thus, it has been hypothesized that stimulation of the medial prefrontal cortex using rTMS may alter the top-down executive control of the DMPFC to striatal regions associated with the urge to binge and purge, thereby improving BN symptoms. In a case report, Downar et al. (Downar, Sankar, Giacobbe, Woodside, \& Colton, 2012)found a dramatic reduction in binge episodes in a woman with severe, refractory $\mathrm{BN}$ who underwent treatment for comorbid depression that targeted the DMPFC. In a subsequent study, the same team reported the outcomes of 28 subjects with refractory binge/purge behaviour who underwent 20-30 sessions of neuronavigated $10-\mathrm{Hz}$ DMPFC rTMS. They reported that 16 of the 28 subjects showed at least 50\% improvement in weekly binge/purge frequency after rTMS. Comparing responders versus non-responders, they found that enhanced frontostriatal connectivity was associated with the responders for binge/purge behaviours. Even though the open design did not allow firm conclusions to be drawn, these studies strongly suggest the interest of further work 
targeting the DMPFC in BN. Other targets have also been explored in the addiction field with promising results: high-frequency stimulation of the superior frontal gyrus(Rose et al., 2011), the anterior insula and the lateral prefrontal cortex using a unique H-coil design (Dinur-Klein et al., 2014) in tobacco use disorder, as well as inhibition of the medial prefrontal cortex in cocaine use disorder using continuous theta burst stimulation (Hanlon et al., 2015).

Transcranial direct current stimulation (tDCS) is another noninvasive brain stimulation method recently explored in the field of addiction, with results similar to those with rTMS(Jansen et al., 2013). A recent review suggested the efficacy of tDCS applied to the DLPFC in reducing food craving (McClelland, Bozhilova, Campbell, \& Schmidt, 2013; Tiffany \& Wray, 2012). Most of the studies included patients with bulimia nervosa, as most participants were defined as healthy subjects with frequent food cravings (Fregni et al., 2008; Goldman et al., 2011; Kekic et al., 2014; Lapenta, Sierve, de Macedo, Fregni, \& Boggio, 2014). Nevertheless, in a recent study, Burgess et al (Burgess et al., 2016)found ameliorating effects of tDCS in a sample of people with binge eating disorders.tDCS might be an alternative to rTMS and merits further assessment in clinical studies.

A notable observation in this study concerns the participants' patterns of response. Although many of the participants showed a marked improvement, a large proportion did not improve or even showed deterioration when exposed to real rTMS. This needs to be put into perspective, given the difficulty in clearly defining the bulimic phenotype. Steiger et al., for example, suggested three subtypes of bulimic disorders (Steiger \& Bruce, 2007): the psychologically intact although perfectionistic type, the overregulated or compulsive type, and the dysregulated or impulsive type. The latter is associated with higher rates of comorbidity, developmental disturbances and possibly also poorer treatment outcome. Very few of the studies thus far (including rTMS) have stratified the subtypes or dimensions of the disorder. We speculate than only certain BN subtypes would benefit from a program of rTMS. 
This is supported by NIMH recognition of EDs as brain-based mental disorders and the subsequent development of the Research Domain Criteria (RDoC)(Chavez \& Insel, 2007; Insel et al., 2010). Some authors (Dunlop, Woodside, \& Downar, 2016; Hanlon et al., 2015) have gone so far as to state that a single 'optimal' protocol for all individuals is highly unlikely and suggest selecting targets based on the neural substrates of phenotypes, as described by the RDoC. The strategies that have been proposed parallel those for substance addiction: damping negative valence systems (prefrontal cortex and insula excitatory stimulation to attenuate amygdala-dependent negative processing), enhancing cognitive/impulse control over urges to binge and purge (excitatory stimulation of the nodes of the salience network, including the DLPFC and insula), or suppressing urge intensity (frontopolar and ventromedial sites inhibitory stimulation).

In conclusion, although rTMS was well-tolerated, we found no evidence that ten sessions of high-frequency rTMS to the left DLPFC provided a greater benefit than placebo in treating bulimic symptoms in a large sample of patients. We strongly encourage future studies to build on these results in order to improve the methodology and investigate alternative designs, samples, and brain targets. 


\section{References}

Ahdab, R., Ayache, S. S., Brugieres, P., Goujon, C., \& Lefaucheur, J. P. (2010). Comparison of "standard" and "navigated" procedures of tms coil positioning over motor, premotor and prefrontal targets in patients with chronic pain and depression. Neurophysiol Clin, 40(1), 27-36. doi: 10.1016/j.neucli.2010.01.001 S09877053(10)00002-X [pii]

Amiaz, R., Levy, D., Vainiger, D., Grunhaus, L., \& Zangen, A. (2009). Repeated highfrequency transcranial magnetic stimulation over the dorsolateral prefrontal cortex reduces cigarette craving and consumption. Addiction, 104(4), 653-660. doi: 10.1111/j.1360-0443.2008.02448.x ADD2448 [pii]

Baeken, C., Vanderhasselt, M. A., Remue, J., Herremans, S., Vanderbruggen, N., Zeeuws, D., ... De Raedt, R. (2013). Intensive hf-rtms treatment in refractory medicationresistant unipolar depressed patients. J Affect Disord, 151(2), 625-631. doi: 10.1016/j.jad.2013.07.008 S0165-0327(13)00561-2 [pii]

Barth, K. S., Rydin-Gray, S., Kose, S., Borckardt, J. J., O'Neil, P. M., Shaw, D., ... George, M. S. (2011). Food cravings and the effects of left prefrontal repetitive transcranial magnetic stimulation using an improved sham condition. Front Psychiatry, 2, 9. doi: 10.3389/fpsyt.2011.00009

Broadbent, H. J., van den Eynde, F., Guillaume, S., Hanif, E. L., Stahl, D., David, A. S., . . . Schmidt, U. (2011). Blinding success of rtms applied to the dorsolateral prefrontal cortex in randomised sham-controlled trials: A systematic review. World J Biol Psychiatry, 12(4), 240-248. doi: 10.3109/15622975.2010.541281

Burgess, E. E., Sylvester, M. D., Morse, K. E., Amthor, F. R., Mrug, S., Lokken, K. L., . . . Boggiano, M. M. (2016). Effects of transcranial direct current stimulation (tdcs) on binge-eating disorder. Int J Eat Disord. doi: 10.1002/eat.22554

Chavez, M., \& Insel, T. R. (2007). Eating disorders: National institute of mental health's perspective. Am Psychol, 62(3), 159-166. doi: 2007-04834-003 [pii] 10.1037/0003-066X.62.3.159

Crow, S. J., Peterson, C. B., Swanson, S. A., Raymond, N. C., Specker, S., Eckert, E. D., \& Mitchell, J. E. (2009). Increased mortality in bulimia nervosa and other eating disorders. Am J Psychiatry, 166(12), 1342-1346. doi: 10.1176/appi.ajp.2009.09020247 appi.ajp.2009.09020247 [pii]

Dinur-Klein, L., Dannon, P., Hadar, A., Rosenberg, O., Roth, Y., Kotler, M., \& Zangen, A. (2014). Smoking cessation induced by deep repetitive transcranial magnetic stimulation of the prefrontal and insular cortices: A prospective, randomized controlled trial. Biol Psychiatry, 76(9), 742-749. doi: 10.1016/j.biopsych.2014.05.020 S0006-3223(14)00387-4 [pii]

Downar, J., Sankar, A., Giacobbe, P., Woodside, B., \& Colton, P. (2012). Unanticipated rapid remission of refractory bulimia nervosa, during high-dose repetitive transcranial magnetic stimulation of the dorsomedial prefrontal cortex: A case report. Front Psychiatry, 3, 30. doi: 10.3389/fpsyt.2012.00030

Dunlop, K. A., Woodside, B., \& Downar, J. (2016). Targeting neural endophenotypes of eating disorders with non-invasive brain stimulation. Front Neurosci, 10, 30. doi: 10.3389/fnins.2016.00030

Eddy, K. T., Dorer, D. J., Franko, D. L., Tahilani, K., Thompson-Brenner, H., \& Herzog, D. B. (2007). Should bulimia nervosa be subtyped by history of anorexia nervosa? A longitudinal validation. Int J Eat Disord, 40 Suppl, S67-71. doi: 10.1002/eat.20422 
Enokibara, M., Trevizol, A., Shiozawa, P., \& Cordeiro, Q. (2016). Establishing an effective tms protocol for craving in substance addiction: Is it possible? Am J Addict, 25(1), 28-30. doi: 10.1111/ajad.12309

Fairburn, C. G., Cooper, Z., \& Shafran, R. (2003). Cognitive behaviour therapy for eating disorders: A "transdiagnostic" theory and treatment. Behav Res Ther, 41(5), 509528. doi: S0005796702000888 [pii]

Fitzgerald, P. B., Hoy, K., Daskalakis, Z. J., \& Kulkarni, J. (2009). A randomized trial of the anti-depressant effects of low- and high-frequency transcranial magnetic stimulation in treatment-resistant depression. Depress Anxiety, 26(3), 229-234. doi: $10.1002 /$ da.20454

Fregni, F., Orsati, F., Pedrosa, W., Fecteau, S., Tome, F. A., Nitsche, M. A., . . Boggio, P. S. (2008). Transcranial direct current stimulation of the prefrontal cortex modulates the desire for specific foods. Appetite, 51(1), 34-41. doi: 10.1016/j.appet.2007.09.016 S0195-6663(07)00429-1 [pii]

Gardner, L. (1991). Edi-2: Professional manual.: Odessa.

Gershon, A. A., Dannon, P. N., \& Grunhaus, L. (2003). Transcranial magnetic stimulation in the treatment of depression. Am J Psychiatry, 160(5), 835-845. doi: 10.1176/appi.ajp.160.5.835

Goldman, R. L., Borckardt, J. J., Frohman, H. A., O'Neil, P. M., Madan, A., Campbell, L. K., ... George, M. S. (2011). Prefrontal cortex transcranial direct current stimulation (tdcs) temporarily reduces food cravings and increases the self-reported ability to resist food in adults with frequent food craving. Appetite, 56(3), 741-746. doi: 10.1016/j.appet.2011.02.013 S0195-6663(11)00096-1 [pii]

Grall-Bronnec, M., \& Sauvaget, A. (2014). The use of repetitive transcranial magnetic stimulation for modulating craving and addictive behaviours: A critical literature review of efficacy, technical and methodological considerations. Neurosci Biobehav Rev, 47, 592-613. doi: S0149-7634(14)00263-2 [pii] 10.1016/j.neubiorev.2014.10.013

Grall-Bronnec, M., Sauvaget, A., Boutin, C., Bulteau, S., Jimenez-Murcia, S., FernandezAranda, F., . . . Caillon, J. (2015). Excessive trading, a gambling disorder in its own right? A case study on a french disordered gamblers cohort. Addict Behav. doi: S0306-4603(15)30072-1 [pii] 10.1016/j.addbeh.2015.12.006

Hadad, N. A., \& Knackstedt, L. A. (2014). Addicted to palatable foods: Comparing the neurobiology of bulimia nervosa to that of drug addiction. Psychopharmacology (Berl), 231(9), 1897-1912. doi: 10.1007/s00213-014-3461-1

Hanlon, C. A., Dowdle, L. T., Austelle, C. W., DeVries, W., Mithoefer, O., Badran, B. W., \& George, M. S. (2015). What goes up, can come down: Novel brain stimulation paradigms may attenuate craving and craving-related neural circuitry in substance dependent individuals. Brain Res, 1628(Pt A), 199-209. doi: 10.1016/j.brainres.2015.02.053 S0006-8993(15)00188-2 [pii]

Hare, T. A., Camerer, C. F., \& Rangel, A. (2009). Self-control in decision-making involves modulation of the vmpfc valuation system. Science, 324(5927), 646-648. doi: 10.1126/science.1168450 324/5927/646 [pii]

Hay, P. P., Bacaltchuk, J., Stefano, S., \& Kashyap, P. (2009). Psychological treatments for bulimia nervosa and binging. Cochrane Database Syst Rev(4), CD000562. doi: 10.1002/14651858.CD000562.pub3

Herremans, S. C., \& Baeken, C. (2012). The current perspective of neuromodulation techniques in the treatment of alcohol addiction: A systematic review. Psychiatr Danub, 24 Suppl 1, S14-20. 
Hone-Blanchet, A., Ciraulo, D. A., Pascual-Leone, A., \& Fecteau, S. (2015). Noninvasive brain stimulation to suppress craving in substance use disorders: Review of human evidence and methodological considerations for future work. Neurosci Biobehav Rev, 59, 184-200. doi: 10.1016/j.neubiorev.2015.10.001 S01497634(15)00253-5 [pii]

Insel, T., Cuthbert, B., Garvey, M., Heinssen, R., Pine, D. S., Quinn, K., . . Wang, P. (2010). Research domain criteria (rdoc): Toward a new classification framework for research on mental disorders. Am J Psychiatry, 167(7), 748-751. doi: 10.1176/appi.ajp.2010.09091379 167/7/748 [pii]

Jansen, J. M., Daams, J. G., Koeter, M. W., Veltman, D. J., van den Brink, W., \& Goudriaan, A. E. (2013). Effects of non-invasive neurostimulation on craving: A meta-analysis. Neurosci Biobehav Rev, 37(10 Pt 2), 2472-2480. doi: 10.1016/j.neubiorev.2013.07.009 S0149-7634(13)00180-2 [pii]

Kekic, M., McClelland, J., Campbell, I., Nestler, S., Rubia, K., David, A. S., \& Schmidt, U. (2014). The effects of prefrontal cortex transcranial direct current stimulation (tdcs) on food craving and temporal discounting in women with frequent food cravings. Appetite, 78, 55-62. doi: 10.1016/j.appet.2014.03.010 S01956663(14)00125-1 [pii]

Klirova, M., Horacek, J., Novak, T., Cermak, J., Spaniel, F., Skrdlantova, L., . . Hoschl, C. (2013). Individualized rtms neuronavigated according to regional brain metabolism ((18)fgd pet) has better treatment effects on auditory hallucinations than standard positioning of rtms: A double-blind, sham-controlled study. Eur Arch Psychiatry Clin Neurosci, 263(6), 475-484. doi: 10.1007/s00406-012-0368-x

Klump, K. L., Bulik, C. M., Kaye, W. H., Treasure, J., \& Tyson, E. (2009). Academy for eating disorders position paper: Eating disorders are serious mental illnesses. Int J Eat Disord, 42(2), 97-103. doi: 10.1002/eat.20589

Lam, R. W., Chan, P., Wilkins-Ho, M., \& Yatham, L. N. (2008). Repetitive transcranial magnetic stimulation for treatment-resistant depression: A systematic review and metaanalysis. Can J Psychiatry, 53(9), 621-631.

Lapenta, O. M., Sierve, K. D., de Macedo, E. C., Fregni, F., \& Boggio, P. S. (2014). Transcranial direct current stimulation modulates erp-indexed inhibitory control and reduces food consumption. Appetite, 83, 42-48. doi: 10.1016/j.appet.2014.08.005 S0195-6663(14)00402-4 [pii]

Lee, J. C., Blumberger, D. M., Fitzgerald, P. B., Daskalakis, Z. J., \& Levinson, A. J. (2012). The role of transcranial magnetic stimulation in treatment-resistant depression: A review. Curr Pharm Des, 18(36), 5846-5852. doi: CPD-EPUB-20120606-6 [pii]

Mantovani, A., Pavlicova, M., Avery, D., Nahas, Z., McDonald, W. M., Wajdik, C. D., . . . Lisanby, S. H. (2012). Long-term efficacy of repeated daily prefrontal transcranial magnetic stimulation (tms) in treatment-resistant depression. Depress Anxiety, 29(10), 883-890. doi: 10.1002/da.21967

McClelland, J., Bozhilova, N., Campbell, I., \& Schmidt, U. (2013). A systematic review of the effects of neuromodulation on eating and body weight: Evidence from human and animal studies. Eur Eat Disord Rev, 21(6), 436-455. doi: 10.1002/erv.2256

McClelland, J., Bozhilova, N., Nestler, S., Campbell, I. C., Jacob, S., Johnson-Sabine, E., \& Schmidt, U. (2013). Improvements in symptoms following neuronavigated repetitive transcranial magnetic stimulation (rtms) in severe and enduring anorexia nervosa: Findings from two case studies. Eur Eat Disord Rev, 21(6), 500506. doi: $10.1002 /$ erv.2266 
McDonald, W. M., Durkalski, V., Ball, E. R., Holtzheimer, P. E., Pavlicova, M., Lisanby, S. H., ... George, M. S. (2011). Improving the antidepressant efficacy of transcranial magnetic stimulation: Maximizing the number of stimulations and treatment location in treatment-resistant depression. Depress Anxiety, 28(11), 973-980. doi: $10.1002 /$ da. 20885

Mitchell, J. E., Myers, T., Crosby, R., O'Neill, G., Carlisle, J., \& Gerlach, S. (2009). Health care utilization in patients with eating disorders. Int J Eat Disord, 42(6), 571-574. doi: 10.1002/eat.20651

Nauczyciel, C., Hellier, P., Morandi, X., Blestel, S., Drapier, D., Ferre, J. C., . . Millet, B. (2011). Assessment of standard coil positioning in transcranial magnetic stimulation in depression. Psychiatry Res, 186(2-3), 232-238. doi: 10.1016/j.psychres.2010.06.012 S0165-1781(10)00354-9 [pii]

Romano, S. J., Halmi, K. A., Sarkar, N. P., Koke, S. C., \& Lee, J. S. (2002). A placebocontrolled study of fluoxetine in continued treatment of bulimia nervosa after successful acute fluoxetine treatment. Am J Psychiatry, 159(1), 96-102. doi: 10.1176/appi.ajp.159.1.96

Rose, J. E., McClernon, F. J., Froeliger, B., Behm, F. M., Preud'homme, X., \& Krystal, A. D. (2011). Repetitive transcranial magnetic stimulation of the superior frontal gyrus modulates craving for cigarettes. Biol Psychiatry, 70(8), 794-799. doi: 10.1016/j.biopsych.2011.05.031 S0006-3223(11)00591-9 [pii]

Rossi, S., Hallett, M., Rossini, P. M., \& Pascual-Leone, A. (2009). Safety, ethical considerations, and application guidelines for the use of transcranial magnetic stimulation in clinical practice and research. Clin Neurophysiol, 120(12), 20082039. doi: 10.1016/j.clinph.2009.08.016 S1388-2457(09)00519-7 [pii]

Sauvaget, A., Trojak, B., Bulteau, S., Jimenez-Murcia, S., Fernandez-Aranda, F., Wolz, I., . . . Grall-Bronnec, M. (2015). Transcranial direct current stimulation (tdcs) in behavioral and food addiction: A systematic review of efficacy, technical, and methodological issues. Front Neurosci, 9, 349. doi: 10.3389/fnins.2015.00349

Schonfeldt-Lecuona, C., Cardenas-Morales, L., Freudenmann, R. W., Kammer, T., \& Herwig, U. (2010). Transcranial magnetic stimulation in depression--lessons from the multicentre trials. Restor Neurol Neurosci, 28(4), 569-576. doi: 10.3233/RNN-2010-0561 70573V03Q1016753 [pii]

Sheehan, D. V., Lecrubier, Y., Sheehan, K. H., Amorim, P., Janavs, J., Weiller, E., ... Dunbar, G. C. (1998). The mini-international neuropsychiatric interview (m.I.N.I.): The development and validation of a structured diagnostic psychiatric interview for dsm-iv and icd-10. J Clin Psychiatry, 59 Suppl 20, 22-33;quiz 34-57.

Steiger, H., \& Bruce, K. R. (2007). Phenotypes, endophenotypes, and genotypes in bulimia spectrum eating disorders. Can J Psychiatry, 52(4), 220-227.

Steinhausen, H. C., \& Weber, S. (2009). The outcome of bulimia nervosa: Findings from one-quarter century of research. Am J Psychiatry, 166(12), 1331-1341. doi: 10.1176/appi.ajp.2009.09040582 appi.ajp.2009.09040582 [pii]

Striegel-Moore, R. H., DeBar, L., Wilson, G. T., Dickerson, J., Rosselli, F., Perrin, N., . . . Kraemer, H. C. (2008). Health services use in eating disorders. Psychol Med, 38(10), 1465-1474. doi: S0033291707001833 [pii] $10.1017 /$ S0033291707001833

Sutoh, C., Koga, Y., Kimura, H., Kanahara, N., Numata, N., Hirano, Y., ... Shimizu, E. (2016). Repetitive transcranial magnetic stimulation changes cerebral oxygenation on the left dorsolateral prefrontal cortex in bulimia nervosa: A near-infrared spectroscopy pilot study. Eur Eat Disord Rev, 24(1), 83-88. doi: 10.1002/erv.2413 
Tiffany, S. T., \& Wray, J. M. (2012). The clinical significance of drug craving. Ann N Y Acad Sci, 1248, 1-17. doi: 10.1111/j.1749-6632.2011.06298.x

Treasure, J., Claudino, A. M., \& Zucker, N. (2010). Eating disorders. Lancet, 375(9714), 583-593. doi: 10.1016/S0140-6736(09)61748-7 S0140-6736(09)61748-7 [pii]

Uher, R., Yoganathan, D., Mogg, A., Eranti, S. V., Treasure, J., Campbell, I. C., ... Schmidt, U. (2005). Effect of left prefrontal repetitive transcranial magnetic stimulation on food craving. Biol Psychiatry, 58(10), 840-842. doi: S0006-3223(05)00706-7 [pii] 10.1016/j.biopsych.2005.05.043

Val-Laillet, D., Aarts, E., Weber, B., Ferrari, M., Quaresima, V., Stoeckel, L. E., . . Stice, E. (2015). Neuroimaging and neuromodulation approaches to study eating behavior and prevent and treat eating disorders and obesity. Neuroimage Clin, 8, 1-31. doi: 10.1016/j.nicl.2015.03.016 S2213-1582(15)00058-3 [pii]

Van den Eynde, F., Claudino, A. M., Mogg, A., Horrell, L., Stahl, D., Ribeiro, W., ... Schmidt, U. (2010). Repetitive transcranial magnetic stimulation reduces cue-induced food craving in bulimic disorders. Biol Psychiatry, 67(8), 793-795. doi: 10.1016/j.biopsych.2009.11.023 S0006-3223(09)01416-4 [pii]

Walpoth, M., Hoertnagl, C., Mangweth-Matzek, B., Kemmler, G., Hinterholzl, J., Conca, A., \& Hausmann, A. (2008). Repetitive transcranial magnetic stimulation in bulimia nervosa: Preliminary results of a single-centre, randomised, double-blind, shamcontrolled trial in female outpatients. Psychother Psychosom, 77(1), 57-60. doi: 000110061 [pii] 10.1159/000110061

Zabala, M. J., Macdonald, P., \& Treasure, J. (2009). Appraisal of caregiving burden, expressed emotion and psychological distress in families of people with eating disorders: A systematic review. Eur Eat Disord Rev, 17(5), 338-349. doi: 10.1002/erv.925 
Table 1: Baseline characteristics of the participants who completed the treatment program

\begin{tabular}{|c|c|c|c|c|c|}
\hline & \multicolumn{2}{|r|}{$\begin{array}{l}\text { Sham } \\
N=24\end{array}$} & \multicolumn{2}{|r|}{$\begin{array}{l}r T M S \\
N=23\end{array}$} & \multirow[b]{2}{*}{$p$} \\
\hline Variable & $n$ & $\%$ & $n$ & $\%$ & \\
\hline Age (years) ${ }^{(1)}$ & \multicolumn{2}{|c|}{$29.50[19-40]$} & \multicolumn{2}{|c|}{$27[19-38]$} & 0.61 \\
\hline Age at the first consultation for EDs (1) & \multicolumn{2}{|c|}{$19[11-36]$} & \multicolumn{2}{|c|}{$19[10-34]$} & 0.63 \\
\hline Previous lifetime history of AN: yes & 12 & 52.17 & 14 & 63.64 & 0.44 \\
\hline Previous hospitalisation for EDs: yes & 11 & 47.83 & 10 & 43.48 & 0.77 \\
\hline Major depressive disorder (lifetime): yes & 18 & 75.00 & 13 & 59.09 & 0.25 \\
\hline Anxiety disorders (lifetime): yes & 7 & 29.17 & 4 & 18.18 & 0.38 \\
\hline Alcohol/substance dependence/abuse: yes & 0 & 0.00 & 4 & 18.18 & 0.05 \\
\hline Depression total score (MADRS)(1) & & $9[3-24]$ & & $11[3-24]$ & 0.94 \\
\hline Number of binge episodes in the last 15 days(1) & & $12.50[0-40]$ & & $0.00[0-14]$ & 0.44 \\
\hline $\begin{array}{l}\text { Number of vomiting episodes in the last } 15 \\
\text { days }^{(1)}\end{array}$ & & $13.00[0-84]$ & & $8.50[0-42]$ & 0.34 \\
\hline \multicolumn{6}{|l|}{ Eating disorders inventory (EDI) dimensions } \\
\hline $\operatorname{Bulimia}^{(1)}$ & \multicolumn{2}{|c|}{$10.00[4.00-17.00]$} & \multicolumn{2}{|c|}{$13.00[5.00-21.00]$} & 0.20 \\
\hline Ineffectiveness $(1)$ & \multicolumn{2}{|c|}{$18.00[8.00-30.00]$} & \multicolumn{2}{|c|}{$22.00[4.00-30.00]$} & 0.23 \\
\hline Perfectionism(1) & \multicolumn{2}{|c|}{$8.00[0.00-15.00]$} & \multicolumn{2}{|c|}{$11.00[1.00-18.00]$} & 0.05 \\
\hline Body Dissatisfaction(1) & \multicolumn{2}{|c|}{$19.00[10.00-24.00]$} & \multicolumn{2}{|c|}{$22.00[9.00-27.00]$} & 0.98 \\
\hline Interpersonal Distrust ${ }^{(1)}$ & \multicolumn{2}{|c|}{$10.00[4.00-20.00]$} & \multicolumn{2}{|c|}{$13.00[4.00-21.00]$} & 0.24 \\
\hline Interoceptive Awareness(1) & \multicolumn{2}{|c|}{$15.00[3.00-28.00]$} & \multicolumn{2}{|c|}{$14.00[5.00-30.00]$} & 0.66 \\
\hline Asceticism(1) & \multicolumn{2}{|c|}{$10.00[4.00-21.00]$} & \multicolumn{2}{|c|}{$10.00[3.00-19.00]$} & 0.81 \\
\hline Impulse Regulation ${ }^{(1)}$ & \multicolumn{2}{|c|}{$5.00[0.00-19.00]$} & \multicolumn{2}{|c|}{$8.00[0.00-29.00]$} & 0.27 \\
\hline Social Insecurity(1) & \multicolumn{2}{|c|}{$15.00[9.00-24.00]$} & \multicolumn{2}{|c|}{$17.00[8.00-24.00]$} & 0.14 \\
\hline Drive for Thinness $(1)$ & \multicolumn{2}{|c|}{$14.00[6.00-21.00]$} & \multicolumn{2}{|c|}{$15.00[2.00-21.00]$} & 0.43 \\
\hline Maturity Fears(1) & \multicolumn{2}{|c|}{$11.00[4.00-21.00]$} & \multicolumn{2}{|c|}{$10.00[1.00-24.00]$} & 0.72 \\
\hline
\end{tabular}

(1) Continuous variables were expressed by median [Minimal value-Maximal value] 
Table 2: Final characteristics of the participants who completed the treatment program

\begin{tabular}{lccc}
\hline & \multicolumn{1}{c}{ Sham } & \multicolumn{1}{c}{ rTMS } \\
& \multicolumn{2}{c}{ N=22 } & N=20 \\
\hline Variable & Median [Min-Max] & Median [Min-Max] & $p$ \\
\hline Number of binge episodes in the last 15 days & $6[0-80]^{*}$ & $7[0-20]^{*}$ & 0.96 \\
\hline Number of vomiting episodes in the last 15 days & $5[0-60]^{*}$ & $7[0-22.50]^{*}$ & 0.50 \\
\hline Maximal craving before a binge episode & $8.5[0-35]$ & $7[0-10]$ & 0.24 \\
\hline MADRS score & $7[1-24]$ & $5[2-18]$ & 0.66 \\
\hline Number of days without binging in the last 15 days & $8[0-15]$ & $8[0-15]$ & 0.86 \\
\hline Length of the longest binge episode in the last 15 days & $60[0-330]$ & $60[0-420]$ & 0.88 \\
\hline
\end{tabular}

* In intention to treat. 


\section{Figure 1: Flowchart of de study}

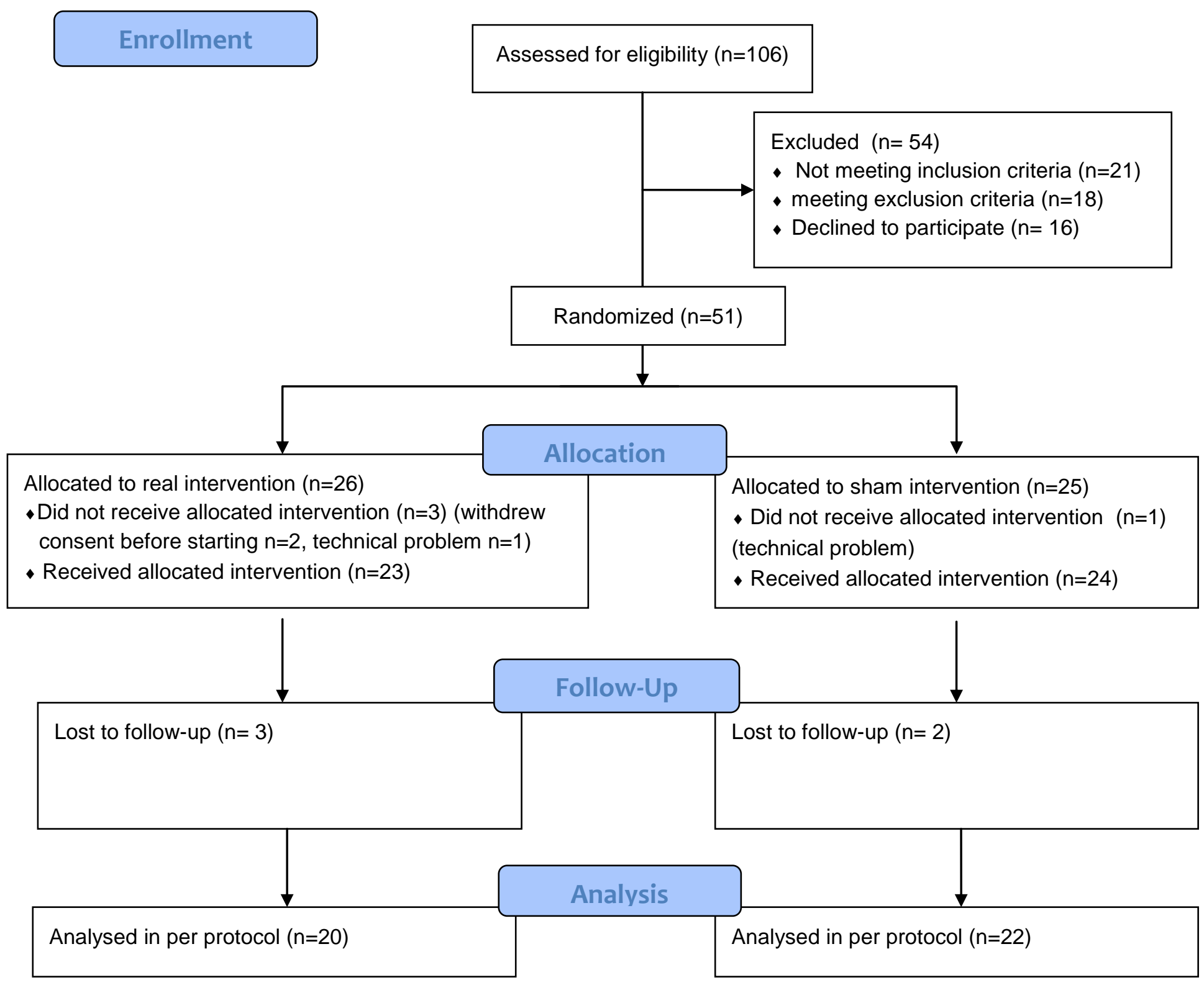



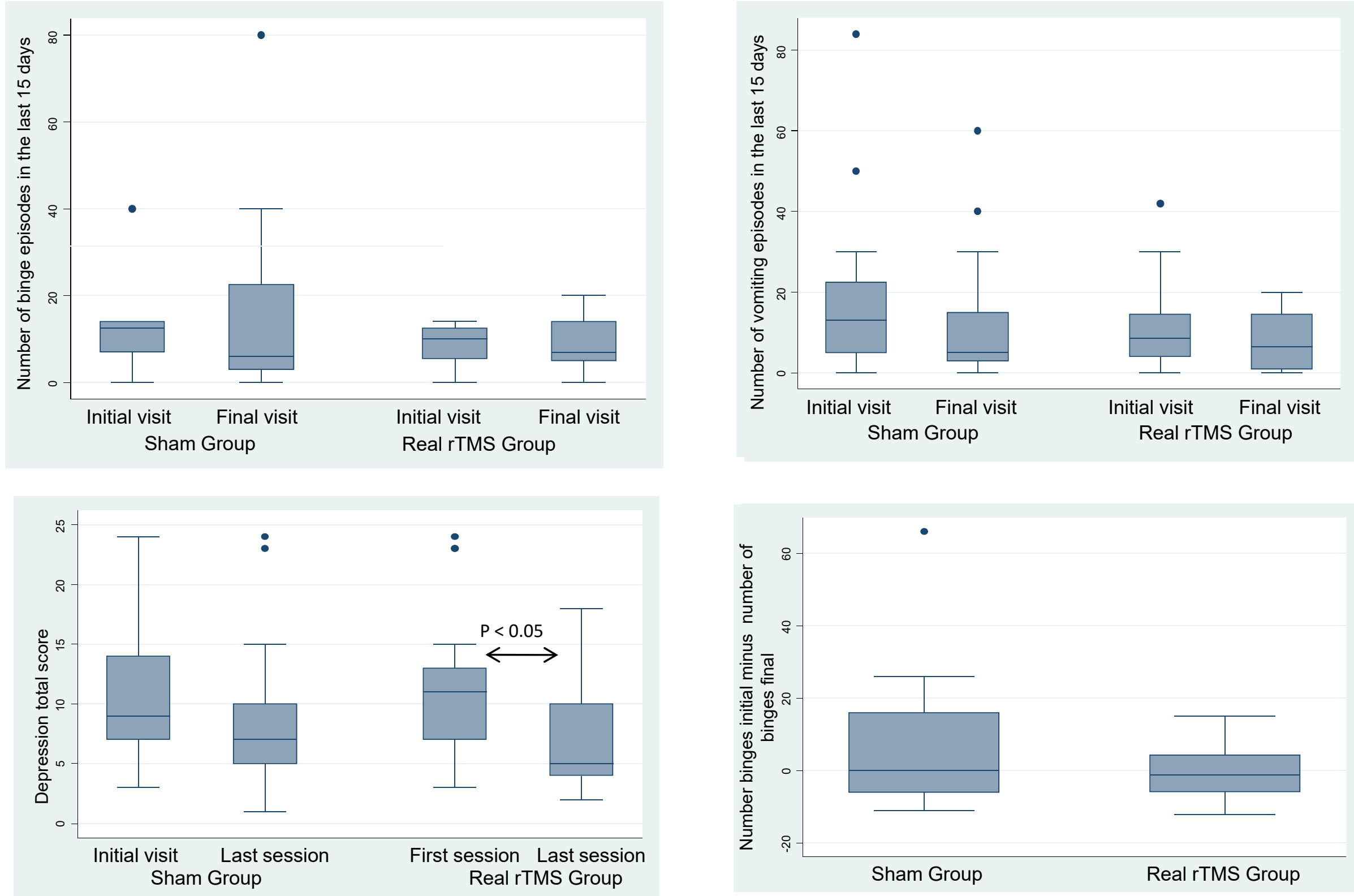

Figure 2: Within-group comparison.

The first 3 graph show changes before after rTMS in each group. The graph number 4 shows the number of binge episodes in the last 15 days at initial visit minus the number of binge episodes in the last 15 days at final visit. Results are shown as a box-whisker plot, with median and 25th quartile 